\title{
PROJECTIVE CURVATURE TENSOR WITH RESPECT TO ZAMKOVOY CONNECTION IN LORENTZIAN PARA-SASAKIAN MANIFOLDS
}

\author{
AbHiJit MANDAL ${ }^{1}$, Ashoke DAS ${ }^{2}$ \\ ${ }^{1}$ Raiganj Surendranath Mahavidyalaya, Raiganj, \\ Uttar Dinajpur, West Bengal, India, \\ Email: abhijit4791@gmail.com \\ ${ }^{2}$ Raiganj University, Raiganj, Uttar Dinajpur, \\ West Bengal, India, \\ Email: ashoke.avik@gmail.com
}

\begin{abstract}
The purpose of the present paper is to study some properties of Projective curvature tensor with respect to Zamkovoy connection in Lorentzian Para Sasakian manifold(briefly, LP-Sasakian manifold). We obtain some results on Lorentzian Para-Sasakian manifold with the help of Zamkovoy connection and Projective curvature tensor. Moreover, we study the LP-Sasakian manifold satisfying $P^{*}(\xi, U) \circ W_{0}^{*}=0$ and $P^{*}(\xi, U) \circ W_{2}^{*}=0$, where $P^{*}, W_{0}^{*}$ and $W_{2}^{*}$ are Projective curvature tensor, $W_{0}$ - curvature tensor and $W_{2}$-curvature tensor with respect to Zamkovoy connection respectively.
\end{abstract}

Key words and Phrases: LP-Sasakian manifolds, Zamkovoy Connection, Projective Curvature tensor

\section{INTRODUCTION}

In 1989, K. Matsumoto [7] first introduced the notion of Lorentzian ParaSasakian manifolds. Also, in 1992, I. Mihai and R. Rosca [8] introduced independently the notion of Lorentzian Para Sasakian manifolds(briefly, LP-Sasakian Manifolds) in classical analysis. In an $n$ - dimensional metric manifold the signature of the metric tensor is the number of positive and negative eigenvalues of the metric. If the metric has $s$ positive eigenvalues and $t$ negative eigenvalues then the signature of the metric is $(s, t)$. For a non-degerate metric tensor $s+t=n$. A Lorentzian manifold is a special case of a semi Riemannian manifold, in which

2020 Mathematics Subject Classification: 53C15, 53C50

Received: 28-05-2020, accepted: 07-10-2020. 
the signature of the metric is $(1, n-1)$ or $(n-1,1)$. And the metric $g$ is called here a Lorentzian metric, which is named after the physicist Hendrik Lorentz. The LP-Sasakian manifold was further studied by several authors. We cite ([3], [9]) and their references.

The notion of Projective curvature tensor was first introduced by K. Yano and S. Bochner [13] in 1953. This curvature tensor was further studied by U. C. De and J. Sengupta [4], S. Ghosh [5]. If there exists a one -one mapping between each co-ordinate neighbourhood of a manifold $M$ to a domain of $R^{n}$ such that any geodesic of $M$ corresponds to a straight line in $R^{n}$, then the manifold $M$ is said to be locally projectively flat. Due to [4], the Projective curvature tensor $P$ of rank four for an $n$-dimensional Riemannian Manifold $M$ is given by

$$
\begin{aligned}
& P(X, Y, Z, V) \\
= & R(X, Y, Z, V)-\frac{1}{n-1}[S(Y, Z) g(X, V)-S(X, Z) g(Y, V)]
\end{aligned}
$$

for all $X, Y, V \& Z \in \chi(M)$, set of all vector fields of the manifold $M$, where $P$ denotes the Projective curvature tensor of type $(0,4)$ and $R$ denotes the Riemannian curvature tensor of type $(0,4)$ defined by

$$
\begin{aligned}
& P(X, Y, Z, V)=g(P(X, Y) Z, V) \\
& R(X, Y, Z, V)=g(R(X, Y) Z, V)
\end{aligned}
$$

where $R$ is the Riemannian curvature tensor of type $(0,3), P$ is the Projective curvature tensor of type $(0,3)$ and $S$ denotes the Ricci tensor of type $(0,2)$.

In 2008, the notion of Zamkovoy connection on para contact manifold was introduced by S. Zamkovoy [14]. Zamkovoy connection was defined as a canonical paracontact connection whose torsion is the obstruction of paracontact manifold to be a para sasakian manifold. This connection was further studied by many researcher. For instance, we see ([2], [1], [6]). For an $n$-dimensional almost contact metric manifold $M$ equipped with an almost contact metric structure $(\phi, \xi, \eta, g)$ consisting of a $(1,1)$ tensor field $\phi$, a vector field $\xi$, a 1-form $\eta$ and a Riemannian metric $g$, the Zamkovoy connection $\left(\nabla^{*}\right)$ in terms of Levi-Civita connection $(\nabla)$ is given by

$$
\nabla_{X}^{*} Y=\nabla_{X} Y+\left(\nabla_{X} \eta\right)(Y) \xi-\eta(Y) \nabla_{X} \xi+\eta(X) \phi Y
$$

for all $X, Y \in \chi(M)$.

In a LP-Sasakian manifold $M$ of dimension $(n>2)$, the Projective curvature tensor $P, W_{0}$ Curvature tensor [10], $W_{2}$-Curvature tensor [12] with respect to the Levi-Civita connection are given by

$$
\begin{gathered}
P(X, Y) Z=R(X, Y) Z-\frac{1}{n-1}[S(Y, Z) X-S(X, Z) Y] \\
W_{0}(X, Y) Z=R(X, Y) Z-\frac{1}{n-1}[S(Y, Z) X-g(X, Z) Q Y] \\
W_{2}(X, Y) Z=R(X, Y) Z-\frac{1}{n-1}[g(Y, Z) Q X-g(X, Z) Q Y]
\end{gathered}
$$


The Projective curvature tensor, $W_{0}$-Curvature tensor and $W_{2}$-Curvature tensor with respect to the Zamkovoy connection are given by,

$$
\begin{gathered}
P^{*}(X, Y) Z=R^{*}(X, Y) Z-\frac{1}{n-1}\left[S^{*}(Y, Z) X-S^{*}(X, Z) Y\right] \\
W_{0}^{*}(X, Y) Z=R^{*}(X, Y) Z-\frac{1}{n-1}\left[S^{*}(Y, Z) X-g(X, Z) Q^{*} Y\right] \\
W_{2}^{*}(X, Y) Z=R^{*}(X, Y) Z-\frac{1}{n-1}\left[g(Y, Z) Q^{*} X-g(X, Z) Q^{*} Y\right]
\end{gathered}
$$

where $R^{*}, S^{*}$ and $Q^{*}$ are Riemannian curvature tensor, Ricci tensor and Ricci operator with respect to Zamkovoy connection $\nabla^{*}$ respectively.

Definition 1.1. An n-dimensional LP-Sasakian manifold $M$ is said to be generalized $\eta$-Einstein manifold if the Ricci tensor of type $(0,2)$ is of the form

$$
S(Y, Z)=k_{1} g(Y, Z)+k_{2} \eta(Y) \eta(Z)+k_{3} \omega(Y, Z)
$$

for all $Y, Z \in \chi(M)$, set of all vector fields of the manifold $M$ and $k_{1}, k_{2}$ and $k_{3}$ are scalars and $\omega$ is a 2 -form.

Definition 1.2. An n-dimensional LP-Sasakian manifold $M$ is said to be Projectively flat if $P(X, Y) Z=0$ for all $X, Y, Z \in \chi(M)$.

Definition 1.3. An n-dimensional LP-Sasakian manifold $M$ is said to be $\xi-$ Projectively flat if $P(X, Y) \xi=0$ for all $X, Y, Z \in \chi(M)$.

This paper is structured as follows: after introduction, a short description of LP-Sasakian manifold is given in section (2). In section (3), we have discussed LP-Sasakian manifold admitting Zamkovoy connection $\nabla^{*}$ and obtain curvature tensor $R^{*}$, Ricci tensor $S^{*}$, Scalar curvature tensor $r^{*}$, in LP-Sasakian manifold. Section (4) contains Projectively flat LP-Sasakian manifold with respect to the connection $\nabla^{*}$. In section (5) we have discussed Locally Projectively $\phi$-symmetric LP-Sasakian manifold $M$ with respect to $\nabla^{*}$. In section (6) we have discussed a LPSasakian manifold satisfying $P^{*}(\xi, U) \circ W_{0}^{*}=0$. In section (7) we have discussed a LP-Sasakian manifold satisfying $P^{*}(\xi, U) \circ W_{2}^{*}=0$.

\section{Preliminaries}

An $n$-dimensional differentiable manifold is called a LP-Sasakian manifold if it admits a $(1,1)$ tensor field $\phi$, a vector field $\xi$, a 1-form $\eta$ and a Lorentzian metric $g$ which satisfies

$$
\begin{aligned}
\phi^{2} Y & =Y+\eta(Y) \xi, \eta(\xi)=-1, \eta(\phi X)=0, \phi \xi=0 \\
g(\phi X, \phi Y) & =g(X, Y)+\eta(X) \eta(Y) \\
g(X, \phi Y) & =g(\phi X, Y), \eta(Y)=g(Y, \xi) \\
\nabla_{X} \xi & =\phi X, g(X, \xi)=\eta(X) \\
\left(\nabla_{X} \phi\right) Y & =g(X, Y) \xi+\eta(Y) X+2 \eta(X) \eta(Y) \xi \\
\forall X, Y & \in \chi(M)
\end{aligned}
$$


where $\nabla$ denotes the operator of covariant differentiation with respect to the Lorentzian metric $g$.

Let us introduced a symmetric $(0,2)$ tensor field $\omega$ such that $\omega(X, Y)=$ $g(X, \phi Y)$. Also, since the vector field $\eta$ is closed in LP-Sasakian manifold, we have

$$
\left(\nabla_{X} \eta\right) Y=\omega(X, Y), \omega(X, \xi)=0, \forall X, Y \in \chi(M)
$$

In LP- Sasakian manifold, the following relations also hold:

$$
\begin{aligned}
& \eta(R(X, Y) Z)=g(Y, Z) \eta(X)-g(X, Z) \eta(Y) \\
& R(X, Y) \xi=\eta(Y) X-\eta(X) Y \\
& R(\xi, Y) Z=g(Y, Z) \xi-\eta(Z) Y \\
& R(\xi, Y) \xi=\eta(Y) \xi+Y \\
& S(X, \xi)=(n-1) \eta(X) \\
& S(\phi X, \phi Y)=S(X, Y)+(n-1) \eta(X) \eta(Y) \\
& Q \xi=(n-1) \xi, Q \phi=\phi Q, S(X, Y)=g(Q X, Y), S^{2}(X, Y)=S(Q X, Y)
\end{aligned}
$$

3. Some Properties of LP-Sasakian manifolds With Respect to ZAMKOVOY CONNECTION

Using (15) and (17) in (4), we get

$$
\nabla_{X}^{*} Y=\nabla_{X} Y+g(X, \phi Y) \xi-\eta(Y) \phi X+\eta(X) \phi Y
$$

with torsion tensor

$$
T^{*}(X, Y)=2[\eta(X) \phi Y-\eta(Y) \phi X]
$$

In view of (4) and (17), we have

$$
\left(\nabla_{X}^{*} g\right)(Y, Z)=-2 g(Y, \phi Z) \eta(X)
$$

Putting $Y=\xi$ in $(25)$

$$
\nabla_{X}^{*} \xi=2 \phi X
$$

Using (14), (15) and (16) in (25), we obtain

$$
\begin{aligned}
& \nabla_{X}^{*}(\phi Y)=\phi\left(\nabla_{X} Y\right)+2 g(X, Y) \xi+\eta(Y) X \\
& +\eta(X) Y+4 \eta(X) \eta(Y) \xi \\
& \nabla_{X}^{*} g(Y, Z)=g\left(\nabla_{X} Y, Z\right)+g\left(Y, \nabla_{X} Z\right) \\
& \nabla_{X}^{*} g(Y, \phi Z)=g\left(\nabla_{X} Y, \phi Z\right)+g\left(Y, \phi \nabla_{X} Z\right)+g(X, Z) \eta(Y) \\
& +g(X, Y) \eta(Z)+2 \eta(X) \eta(Y) \eta(Z)
\end{aligned}
$$


In view of (25), (29), (30) and (31), we have

$$
\begin{aligned}
\nabla_{X}^{*} \nabla_{Y}^{*} Z= & \nabla_{X} \nabla_{Y} Z+g\left(X, \phi \nabla_{Y} Z\right) \xi-\eta\left(\nabla_{Y} Z\right) \phi X+\eta(X) \phi \nabla_{Y} Z \\
& +g\left(\nabla_{X} Y, \phi Z\right) \xi+g\left(Y, \phi \nabla_{X} Z\right) \xi+g(X, Z) \eta(Y) \xi \\
& +g(X, Y) \eta(Z) \xi+2 \eta(X) \eta(Y) \eta(Z) \xi+2 g(Y, \phi Z) \phi X \\
& -g(X, \phi Z) \phi Y-\eta\left(\nabla_{X} Z\right) \phi Y-\phi\left(\nabla_{X} Y\right) \eta(Z)-2 g(X, Y) \eta(Z) \xi \\
& -\eta(Y) \eta(Z) X-\eta(X) \eta(Z) Y-4 \eta(X) \eta(Y) \eta(Z) \xi \\
& +g(X, \phi Y) \phi Z+\eta\left(\nabla_{X} Y\right) \phi Z+\phi\left(\nabla_{X} Z\right) \eta(Y)+2 g(X, Z) \eta(Y) \xi \\
& +\eta(Y) \eta(Z) X+\eta(X) \eta(Y) Z+4 \eta(X) \eta(Y) \eta(Z) \xi
\end{aligned}
$$

Interchanging $X$ and $Y$

$$
\begin{aligned}
\nabla_{Y}^{*} \nabla_{X}^{*} Z= & \nabla_{Y} \nabla_{X} Z+g\left(Y, \phi \nabla_{X} Z\right) \xi-\eta\left(\nabla_{X} Z\right) \phi Y+\eta(Y) \phi \nabla_{X} Z \\
& +g\left(\nabla_{Y} X, \phi Z\right) \xi+g\left(X, \phi \nabla_{Y} Z\right) \xi+g(Y, Z) \eta(X) \xi \\
& +g(Y, X) \eta(Z) \xi+2 \eta(Y) \eta(X) \eta(Z) \xi+2 g(X, \phi Z) \phi Y \\
& -g(Y, \phi Z) \phi X-\eta\left(\nabla_{Y} Z\right) \phi X-\phi\left(\nabla_{Y} X\right) \eta(Z)-2 g(Y, X) \eta(Z) \xi \\
& -\eta(X) \eta(Z) Y-\eta(Y) \eta(Z) X-4 \eta(Y) \eta(X) \eta(Z) \xi \\
& +g(Y, \phi X) \phi Z+\eta\left(\nabla_{Y} X\right) \phi Z+\phi\left(\nabla_{Y} Z\right) \eta(X)+2 g(Y, Z) \eta(X) \xi \\
& +\eta(X) \eta(Z) Y+\eta(Y) \eta(X) Z+4 \eta(Y) \eta(X) \eta(Z) \xi
\end{aligned}
$$

Also we have

$$
\begin{aligned}
\nabla_{[X, Y]}^{*} Z= & \nabla_{[X, Y]} Z+g\left(\nabla_{X} Y, \phi Z\right) \xi-g\left(\nabla_{Y} X, \phi Z\right) \xi-\eta(Z) \phi \nabla_{X} Y \\
& +\eta(Z) \phi \nabla_{Y} X+\eta\left(\nabla_{X} Y\right) \phi Z-\eta\left(\nabla_{Y} X\right) \phi Z
\end{aligned}
$$

Let $R^{*}$ be the Riemannian curvature tensor with respect to Zamkovoy connection and it is defined as

$$
R^{*}(X, Y) Z=\nabla_{X}^{*} \nabla_{Y}^{*} Z-\nabla_{Y}^{*} \nabla_{X}^{*} Z-\nabla_{[X, Y]}^{*} Z
$$

Using (25), (32), (33) and (34) in (35), we get

$$
\begin{aligned}
& R^{*}(X, Y) Z \\
= & R(X, Y) Z+3 g(X, Z) \eta(Y) \xi-3 g(Y, Z) \eta(X) \xi+3 g(Y, \phi Z) \phi X \\
& -3 g(X, \phi Z) \phi Y-\eta(X) \eta(Z) Y+\eta(Y) \eta(Z) X
\end{aligned}
$$

Consequently one can easily bring out the followings:

$$
\begin{aligned}
S^{*}(Y, Z) & =S(Y, Z)+(n-1) \eta(Y) \eta(Z)+3 \psi g(Y, \phi Z) \\
S^{*}(\xi, Z) & =S^{*}(Z, \xi)=0 \\
Q^{*} Y & =Q Y+(n-1) \eta(Y) \xi+3 \psi \phi Y \\
Q^{*} \xi & =0 \\
r^{*} & =r-n+1+3 \psi^{2}
\end{aligned}
$$




$$
\begin{aligned}
& R^{*}(X, Y) \xi=0 \\
& R^{*}(\xi, Y) Z=4 g(\phi Y, \phi Z) \xi \\
& R^{*}(X, \xi) Z=-4 g(\phi X, \phi Z) \xi
\end{aligned}
$$

for all $X, Y, Z \in \chi(M)$, where $\psi=\operatorname{trace}(\phi)$

Thus we can state the followings:

Proposition 3.1. Let $M$ be an n-dimensional LP-Sasakian manifold admitting Zamkovoy connection $\nabla^{*}$, then

(i) The curvature tensor $R^{*}$ of $\nabla^{*}$ is given by (36)

(ii) The Ricci tensor $S^{*}$ of $\nabla^{*}$ is given by (37)

(iii) The scalar curvature $r^{*}$ of $\nabla^{*}$ is given by (41)

(iv) The Ricci tensor $S^{*}$ of $\nabla^{*}$ is symmetric.

(v) $R^{*}$ satisfies: $R^{*}(X, Y) Z+R^{*}(Y, Z) X+R^{*}(Z, X) Y=0$.

\section{Projectively flat LP-Sasakian manifold with Respect to the ZAMKOVOY CONNECTION}

Theorem 4.1. If an $n$-dimensional LP-Sasakian manifold $M$ is Projectively flat with respect to Zamkovoy connection, then it is a generalized $\eta$-Einstein manifold.

Proof. In view of (8), (36) and (37), the Projective curvature tensor $P^{*}$ with respect to the Zamkovoy connection $\nabla^{*}$ on a LP-Sasakian manifold $M$ of dimension $(n>2)$ takes the form

$$
\begin{aligned}
P^{*}(X, Y) Z= & R(X, Y) Z+3 g(X, Z) \eta(Y) \xi-3 g(Y, Z) \eta(X) \xi+3 g(Y, \phi Z) \phi X \\
& -3 g(X, \phi Z) \phi Y-\eta(X) \eta(Z) Y+\eta(Y) \eta(Z) X \\
& -\frac{1}{n-1}[S(Y, Z) X+(n-1) \eta(Y) \eta(Z) X+3 \psi g(Y, \phi Z) X] \\
& +\frac{1}{n-1}[S(X, Z) Y+(n-1) \eta(X) \eta(Z) Y+3 \psi g(X, \phi Z) Y](45)
\end{aligned}
$$

Let $M$ be projectively flat with respect to Zamkovoy connection, then from (45), we get

$$
\begin{aligned}
& R(X, Y) Z \\
= & -3 g(X, Z) \eta(Y) \xi+3 g(Y, Z) \eta(X) \xi-3 g(Y, \phi Z) \phi X \\
& +3 g(X, \phi Z) \phi Y+\eta(X) \eta(Z) Y-\eta(Y) \eta(Z) X \\
& +\frac{1}{n-1}[S(Y, Z) X+(n-1) \eta(Y) \eta(Z) X+3 \psi g(Y, \phi Z) X] \\
& -\frac{1}{n-1}[S(X, Z) Y+(n-1) \eta(X) \eta(Z) Y+3 \psi g(X, \phi Z) Y]
\end{aligned}
$$


Taking inner product of (46) with a vector field $V$, we have

$$
\begin{aligned}
& R(X, Y, Z, V) \\
= & -3 g(X, Z) \eta(Y) \eta(V)+3 g(Y, Z) \eta(X) \eta(V)-3 g(Y, \phi Z) g(\phi X, V) \\
& +3 g(X, \phi Z) g(\phi Y, V)+\eta(X) \eta(Z) g(Y, V)-\eta(Y) \eta(Z) g(X, V) \\
& +\frac{1}{n-1}[S(Y, Z)+(n-1) \eta(Y) \eta(Z)+3 \psi g(Y, \phi Z)] g(X, V) \\
& -\frac{1}{n-1}[S(X, Z)+(n-1) \eta(X) \eta(Z)+3 \psi g(X, \phi Z)] g(Y, V)
\end{aligned}
$$

Setting $X=V=\xi$ and using (12), (22) in (47), we get

$$
S(Y, Z)=4(n-1) g(Y, Z)+3(n-1) \eta(Y) \eta(Z)-3 \psi \omega(Y, Z)
$$

where $\omega(Y, Z)=g(\phi Y, Z)$.

which shows that $M$ is an $\eta$-Einstein manifold. Hence the theorem is proved.

Corollary 4.2. An $n$ - dimensional LP-Sasakian manifold $M$ is $\xi$ - Projectively flat with respect to Zamkovoy connection iff it is so with respect to Levi-Civita connection.

Proof. Using (5) in (45), we get

$$
\begin{aligned}
P^{*}(X, Y) Z= & P(X, Y) Z+3 g(X, Z) \eta(Y) \xi-3 g(Y, Z) \eta(X) \xi \\
& +3 g(Y, \phi Z) \phi X-3 g(X, \phi Z) \phi Y \\
& -\frac{3 \psi}{n-1}[g(Y, \phi Z) X+g(X, \phi Z) Y]
\end{aligned}
$$

Setting $Z=\xi$ in (48), we get

$$
P^{*}(X, Y) \xi=P(X, Y) \xi
$$

Therefore, $M$ is $\xi$-Projectively flat with respect to Zamkovoy connection iff it is so with respect to Levi-Civita connection.

\section{Locally Projectively $\phi$-Symmetric LP-SASAKIAN MANifoldS With RESPECT TO ZAMKOVOY CONNECTION}

In 1977, Takahashi [11] first studied the concept of locally $\phi$-symmetry on Sasakian manifold. In this section we consider a locally projectively $\phi$-symmetric LP-Sasakian manifolds with respect to the connection $\nabla^{*}$.

Definition 5.1. An n-dimensional LP-Sasakian manifold $M$ is said to be locally projectively $\phi$-symmetric with respect to Zamkovoy connection $\nabla^{*}$ if the projective curvature tensor $P^{*}$ with respect to the connection $\nabla^{*}$ satisfies

$$
\phi^{2}\left(\nabla_{W}^{*} P^{*}\right)(X, Y) Z=0
$$

where $X, Y, Z$ and $W$ are horizontal vector fields on $M$, i.e $X, Y, Z$ and $W$ are orthonormal to $\xi$ on the manifold $M$. 
Theorem 5.2. An $n$-dimensional LP-Sasakian manifold $M(n>3)$ is locally projectively $\phi$-symmetric with respect to Zamkovoy connection if and only if it is so with respect to the Levi-Civita connection, provided trace $(\phi)=0$.

Proof. In view of (25), we have

$$
\begin{aligned}
\left(\nabla_{W}^{*} P^{*}\right)(X, Y) Z= & \left(\nabla_{W} P^{*}\right)(X, Y) Z+g\left(W, \phi P^{*}(X, Y) Z\right) \xi \\
& -\eta\left(P^{*}(X, Y) Z\right) \phi W+\eta(W) \phi P^{*}(X, Y) Z
\end{aligned}
$$

Taking covariant differentiation of (48) in the direction of $W$ and considering $\operatorname{trace}(\phi)=0$, we obtain

$$
\begin{aligned}
\left(\nabla_{W} P^{*}\right)(X, Y) Z= & \left(\nabla_{W} P\right)(X, Y) Z \\
& +3[g(X, Z) g(W, \phi Y)-g(Y, Z) g(W, \phi X)] \xi \\
& +3[g(W, Z) \eta(Y)+g(Y, W) \eta(Z)+2 \eta(W) \eta(Y) \eta(Z)] \phi X \\
& +3 g(Y, \phi Z)[g(W, X) \xi+\eta(X) W+2 \eta(W) \eta(X) \xi] \\
& -3[g(W, Z) \eta(X)+g(X, W) \eta(Z)+2 \eta(W) \eta(X) \eta(Z)] \phi Y \\
& -3 g(X, \phi Z)[g(W, Y) \xi+\eta(Y) W+2 \eta(W) \eta(Y) \xi]
\end{aligned}
$$

In view of $(12),(18)$ and $(45)$, we obtain

$$
\begin{aligned}
& \eta\left(P^{*}(X, Y) Z\right) \\
= & g(Y, Z) \eta(X)-g(X, Z) \eta(Y)-3 g(X, Z) \eta(Y)+3 g(Y, Z) \eta(X) \\
& -\frac{1}{n-1}[S(Y, Z)+(n-1) \eta(Y) \eta(Z)+3 \psi g(Y, \phi Z)] \eta(X) \\
& +\frac{1}{n-1}[S(X, Z)+(n-1) \eta(X) \eta(Z)+3 \psi g(X, \phi Z)] \eta(Y)
\end{aligned}
$$

Using (51) and (52) in (50), we get

$$
\begin{aligned}
\left(\nabla_{W}^{*} P^{*}\right)(X, Y) Z= & \left(\nabla_{W} P\right)(X, Y) Z+3[g(X, Z) g(W, \phi Y)-g(Y, Z) g(W, \phi X)] \xi \\
& +3[g(W, Z) \eta(Y)+g(Y, W) \eta(Z)+2 \eta(W) \eta(Y) \eta(Z)] \phi X \\
& +3 g(Y, \phi Z)[g(W, X) \xi+\eta(X) W+2 \eta(W) \eta(X) \xi] \\
& -3[g(W, Z) \eta(X)+g(X, W) \eta(Z)+2 \eta(W) \eta(X) \eta(Z)] \phi Y \\
& -3 g(X, \phi Z)[g(W, Y) \xi+\eta(Y) W+2 \eta(W) \eta(Y) \xi] \\
& +g\left(W, \phi P^{*}(X, Y) Z\right) \xi-g(Y, Z) \eta(X) \phi W+g(X, Z) \eta(Y) \phi W \\
& +3 g(X, Z) \eta(Y) \phi W-3 g(Y, Z) \eta(X) \phi W \\
& +\frac{1}{n-1}[S(Y, Z)+(n-1) \eta(Y) \eta(Z)+3 \psi g(Y, \phi Z)] \eta(X) \phi W \\
& -\frac{1}{n-1}[S(X, Z)+(n-1) \eta(X) \eta(Z)+3 \psi g(X, \phi Z)] \eta(Y) \phi W \\
& +\phi P(X, Y) Z \eta(W)+3 g(Y, \phi Z) X \eta(W)-3 g(X, \phi Z) Y \eta(W) \\
& +3 g(Y, \phi Z) \eta(X) \eta(W) \xi-3 g(X, \phi Z) \eta(Y) \eta(W) \xi
\end{aligned}
$$

Applying $\phi^{2}$ on both sides of (53) and using (12), we obtain

$$
\phi^{2}\left(\nabla_{W}^{*} P^{*}\right)(X, Y) Z=\phi^{2}\left(\nabla_{W} P\right)(X, Y) Z
$$


where $X, Y, Z, W$ are horizontal vector fields and $\operatorname{trace}(\phi)=0$. Hence the theorem is proved.

6. LP-Sasakian MANifold SATISFying $P^{*}(\xi, U) \circ W_{0}^{*}=0$

Theorem 6.1. In an $n$-dimensional $(n>3)$ LP-Sasakian manifold $M$ admitting Zamkovoy connection $\nabla^{*}$, if the condition $P^{*}(\xi, U) \circ W_{0}^{*}=0$ holds, then the equation

$$
\begin{aligned}
S^{2}(X, Y)= & 4(n-1) S(X, Y)-6 \psi S(\phi X, Y)+12(n-1) \psi g(X, \phi Y) \\
& -9 \psi^{2} g(X, Y)+\left[3(n-1)^{2}-9 \psi^{2}\right] \eta(X) \eta(Y)
\end{aligned}
$$

is satisfied on the manifold $M$, for all $X, Y \in \chi(M)$.

Proof. It can be easily seen from (8) and (9), that

$$
\begin{gathered}
P^{*}(\xi, U) X=4 g(\phi U, \phi X) \xi-\frac{1}{n-1} S^{*}(U, X) \xi \\
P^{*}(\xi, Y) \xi=0, P^{*}(\xi, \xi) Y=0, P^{*}(X, Y) \xi=0 \\
W_{0}^{*}(X, Y) \xi=\frac{1}{n-1} \eta(X) Q^{*} Y, W_{0}^{*}(\xi, Y) \xi=-\frac{1}{n-1} Q^{*} Y
\end{gathered}
$$

Let us consider a LP- Sasakian manifold $M$ satisfying the condition

$$
\left(P^{*}(\xi, U) \circ W_{0}^{*}\right)(X, Y) Z=0 .
$$

Then we have

$$
\begin{aligned}
0= & P^{*}(\xi, U) W_{0}^{*}(X, Y) Z-W_{0}^{*}\left(P^{*}(\xi, U) X, Y\right) Z \\
& -W_{0}^{*}\left(X, P^{*}(\xi, U) Y\right) Z-W_{0}^{*}(X, Y) P^{*}(\xi, U) Z
\end{aligned}
$$

Replacing $Z$ by $\xi$ in (58), we get

$$
\begin{aligned}
0= & P^{*}(\xi, U) W_{0}^{*}(X, Y) \xi-W_{0}^{*}\left(P^{*}(\xi, U) X, Y\right) \xi \\
& -W_{0}^{*}\left(X, P^{*}(\xi, U) Y\right) \xi-W_{0}^{*}(X, Y) P^{*}(\xi, U) \xi
\end{aligned}
$$

Using (55), (56) and (57) in (59), we have

$$
\begin{aligned}
0= & 4 S^{*}(\phi U, \phi Y) \eta(X) \xi-\frac{1}{n-1} S^{*}\left(U, Q^{*} Y\right) \eta(X) \xi \\
& +4 g(\phi U, \phi X) Q^{*} Y-\frac{1}{n-1} S^{*}(U, X) Q^{*} Y
\end{aligned}
$$

The inner product of the equation (60) with vector field $V$ gives

$$
\begin{aligned}
0= & 4 S^{*}(\phi U, \phi Y) \eta(X) \eta(V)-\frac{1}{n-1} S^{*}\left(U, Q^{*} Y\right) \eta(X) \eta(V) \\
& +4 g(\phi U, \phi X) S^{*}(Y, V)-\frac{1}{n-1} S^{*}(U, X) S^{*}(Y, V)
\end{aligned}
$$

Let $\left\{e_{i}\right\}(1 \leq i \leq n)$ be an orthonormal basis of the tangent space at any point of the manifold $M$. Setting $U=V=e_{i}$ and taking summation over $i(1 \leq i \leq n)$ and using (23), (24), (37), (38) and (39) in (61), we get 


$$
\begin{aligned}
S^{2}(X, Y)= & 4(n-1) S(X, Y)-6 \psi S(\phi X, Y)+12(n-1) \psi g(X, \phi Y) \\
& -9 \psi^{2} g(X, Y)+\left[3(n-1)^{2}-9 \psi^{2}\right] \eta(X) \eta(Y)
\end{aligned}
$$

Hence the theorem is proved.

\section{LP-SASAKIAN MANifold SATISFYing $P^{*}(\xi, U) \circ W_{2}^{*}=0$}

Theorem 7.1. In an $n$-dimensional LP-Sasakain manifold $M$ of dimension $(n>3)$ if the condition $P^{*}(\xi, U) \circ W_{2}^{*}=0$ holds, then the equation

$$
\begin{aligned}
S^{2}(X, Z)= & 4(n-1) S(X, Z)-6 \psi S(X, \phi Z)+12(n-1) \psi g(X, \phi Z) \\
& -9 \psi^{2} g(X, Z)+\left[3(n-1)^{2}-9 \psi^{2}\right] \eta(X) \eta(Z)
\end{aligned}
$$

is satisfied on $M$, for all $X, Z \in \chi(M)$.

Proof. Let us consider a LP- Sasakian manifold $M$ satisfying the condition

$$
\left(P^{*}(\xi, U) \circ W_{2}^{*}\right)(X, Y) Z=0
$$

Then we have

$$
\begin{aligned}
0= & P^{*}(\xi, U) W_{2}^{*}(X, Y) Z-W_{2}^{*}\left(P^{*}(\xi, U) X, Y\right) Z \\
& -W_{2}^{*}\left(X, P^{*}(\xi, U) Y\right) Z-W_{2}^{*}(X, Y) P^{*}(\xi, U) Z
\end{aligned}
$$

Replacing $Y$ by $\xi$ in (64), we get

$$
\begin{aligned}
0= & P^{*}(\xi, U) W_{2}^{*}(X, \xi) Z-W_{2}^{*}\left(P^{*}(\xi, U) X, \xi\right) Z \\
& -W_{2}^{*}\left(X, P^{*}(\xi, U) \xi\right) Z-W_{2}^{*}(X, \xi) P^{*}(\xi, U) Z
\end{aligned}
$$

It is seen that

$$
\begin{aligned}
W_{2}^{*}(X, \xi) Z & =-4 g(\phi X, \phi Z) \xi-\frac{1}{n-1} \eta(Z) Q^{*} X \\
W_{2}^{*}(\xi, \xi) Z & =0, W_{2}^{*}(X, \xi) \xi=\frac{1}{n-1} Q^{*} X
\end{aligned}
$$

Using (55), (56), (66) and (67) in (65), we get

$$
\begin{aligned}
0= & \eta(Z) 4 g\left(\phi U, \phi Q^{*} X\right) \xi-\frac{1}{n-1} \eta(Z) S^{*}\left(U, Q^{*} X\right) \xi \\
& 4 g(\phi U, \phi Z) Q^{*} X-\frac{1}{n-1} S^{*}(U, Z) Q^{*} X
\end{aligned}
$$

The inner product of the equation (68) with vector field $V$ gives

$$
\begin{aligned}
0= & {\left[4 S^{*}(\phi U, \phi X)-\frac{1}{n-1} S^{*}\left(U, Q^{*} X\right)\right] \eta(Z) \eta(V) } \\
& +\left[4 g(\phi U, \phi Z)-\frac{1}{n-1} S^{*}(U, Z)\right] S^{*}(X, V)
\end{aligned}
$$


Let $\left\{e_{i}\right\}(1 \leq i \leq n)$ be an orthonormal basis of the tangent space at any point of the manifold $M$. Setting $U=V=e_{i}$ and taking summation over $i(1 \leq i \leq n)$ and using (23), (24), (37), (38) and (39) in (69), we get

$$
\begin{aligned}
0= & 4(n-1) S(X, Z)-6 \psi S(X, \phi Z)+12(n-1) \psi g(X, \phi Z) \\
& -9 \psi^{2} g(X, Z)+\left[3(n-1)^{2}-9 \psi^{2}\right] \eta(X) \eta(Z)-S^{2}(X, Z)
\end{aligned}
$$

Using (36) in (70), we have

$$
\begin{aligned}
S^{2}(X, Z)= & 4(n-1) S(X, Z)-6 \psi S(X, \phi Z)+12(n-1) \psi g(X, \phi Z) \\
& -9 \psi^{2} g(X, Z)+\left[3(n-1)^{2}-9 \psi^{2}\right] \eta(X) \eta(Z)
\end{aligned}
$$

Hence the theorem is proved.

Acknowledgement. The authors would like to thank the referee for their valuable suggestions to improve the paper.

\section{REFERENCES}

[1] Biswas, A. and Baishya, K. K., "Study on generalized pseudo (Ricci) symmetric Sasakian manifold admitting general connection", Bulletin of the Transilvania University of Brasov, 12(2) (2020) 233-246.

[2] Blaga, A. M., "Canonical connection on Para Kenmotso manifold", Novi Sad . J. Math, Vol 45, No.2 (2015) 131-142.

[3] De, U. C. and Matsumoto, K. and Shaikh, A. A., "On Lorentzian para-Sasakian manifolds", Rendiconti del Seminario Matematico di Messina, Serie II, Supplemento al n. 3(1999), 149158.

[4] De, U. C. and Sengupta, J., "On a Type of SemiSymmetric Metric Connection on an almostcontact metric connection", Facta Universitatis Ser. Math. Inform. 16(2001) 87-96.

[5] Ghosh, S., "On a class of $(k, \mu)$ - contact manifolds", Bull. Cal. Math. Soc. 102 (2010), 219-226.

[6] Mandal, A. and Das, A., "On M-Projective Curvature Tensor of Sasakian Manifolds admitting Zamkovoy Connection", Adv. Math. Sci. J, 9(2020), no.10, 8929-8940.

[7] Matsumoto, K., "On Lorentzian paracontact manifolds", Bull. of Yamagata Univ., Nat. Sci.12 (1989), p151-156.

[8] Mihai, I. and Rosca, R., "On Lorentzian P-Sasakian manifolds, Classical Analysis", World Scientific Publi. (1992), 155-169.

[9] Ozgur, C., " $\varphi$-Conformally flat Lorentzian para Saskian manifolds", Radovi Mathemeticki,Vol(12), (2003), p-99-106.

[10] Pokhariyal, G. P. and Mishra, R. S., "Curvature tensors and their relativistic signifcance", Yokohama Math. J., 18(1970), 105-108.

[11] Takahashi, T., "Sasakian $\phi$-symmetric spaces", Tohoku Mathematical Journal, Second Series, vol. 29, no.1 (1977), pp. 91-113.

[12] Tripathi, M. M. and Gupta. P., "On $\tau$ - curvature tensor in K-contact manifold and Sasakian manifold", International Electronic Journal of Mathematics,V-04,(2011), p32-47.

[13] Yano, K. and Bochner, S., "Curvature and Betti numbers", Annals of Mathematics Studies 32, Princeton University Press (1953).

[14] Zamkovoy, S., "Canonical connections on paracontact manifolds", Ann. Global Anal. Geom. $36(1)(2008), 37-60$. 